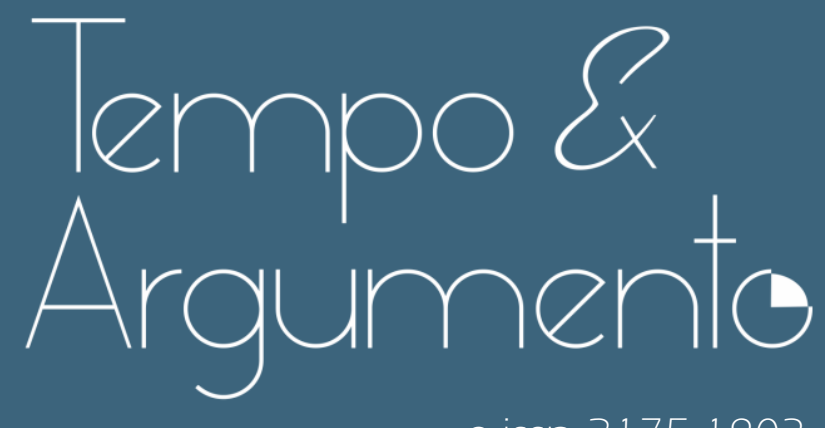

e-issn 2175-1803

\title{
Russell, crítico da ditadura militar brasileira
}

- Murilo Garcia de Matos Amaral

Doutor em Filosofia pela Universidade Federal da Bahia (UFBA).

Membro do grupo Empirismo, Fenomenologia e Gramática da

Universidade Federal da Bahia (EFG/UFBA).

Salvador, BA - BRASIL

lattes.cnpq.br/4754821844867329

murilogmamaral@gmail.com

(iD) orcid.org/0000-0002-2766-091X

Para citar este texto:

AMARAL, Murilo Garcia de Matos. Russell, crítico da ditadura militar brasileira

[Fontes do Tempo Presente]. Revista Tempo e Argumento, Florianópolis, v. 12, n.

30, e050 1. maio/ago. 2020.

doi dx.doi.org/10.5965/2175180312302020e050 1

Recebido: 21/01/2020

Aprovado: 24/04/2020 
Bertrand Russell teve uma contribuição póstuma importante para o enfrentamento à ditadura militar no Brasil, com o legado deixado pelo Russell Tribunal. Esse legado está historiado no documento "Brasil, violação dos direitos humanos - Tribunal Russell II", que foi reeditado e publicado pela Editora UFPB em 20141. O que não se sabia até então é que, em vida, Russell se posicionou contra a prisão de políticos de esquerda e intelectuais perante as autoridades brasileiras um mês após o golpe de 1964. Além disso, entre 1964 e 1966, ele manteve contato com brasileiros, tendo recebido inúmeras denúncias de violações de direitos humanos, e colocou a Bertrand Russell Peace Foundation ${ }^{2}$ a postos para investigar e tornar público o que se passava no país. A seguir, apresento algumas das cartas que relatam esses fatos, descobertas em 2016, na ocasião de minha visita ao Bertrand Russell Archives ${ }^{3}$.

\section{Uma carta "não enviada" a João Goulart}

Em 12 de fevereiro de 1964, Russell escreveu uma carta a João Goulart dizendo que ouviu falar do interesse do presidente por assuntos internacionais e de sua preocupação com "o bem-estar de sua gente”. Ele propõe o envio de dois representantes ao Brasil para discutir os trabalhos da BRPF e formar "uma associação pública”. A carta não foi enviada (nos registros, está riscada com um "not sent"). Por isso, diferentemente dos outros registros de cartas, que são cópias carbono, este tem a assinatura de Russell. A razão da carta não ter sido enviada é desconhecida. Também não se sabe o quanto Russell estava a par das tensões políticas no Brasil na época, nem o que exatamente o levou a (quase) buscar uma aproximação com João Goulart naquele momento.

\footnotetext{
'Este documento é uma reedição do original italiano de 1975. Ele traz os depoimentos realizados na sessão do Tribunal Russell II destinada ao Brasil. Encontra-se no site do Ministério da Justiça, na seção "Anistia Política”. TOSI, Giuseppe (org.); FERREIRA, Lúcia de Fátima Guerra (org.). Brasil, violação dos direitos humanos - Tribunal Russell II. João Pessoa: Editora UFPB, 2014. Disponível em: $\quad$ <http://www.justica.gov.br/central-de-conteudo/anistia/anexos/brasil_violacao-direitoshumanos-miolo-final.pdf>.

2 Fundada em 1963, a Bertrand Russell Peace Foundation (doravante BRPF) buscou impulsionar o ativismo de Russell em prol dos direitos humanos e da justiça social, e, especialmente, contra a produção de armas nucleares.

${ }^{3}$ Agradeço ao Governo do Canadá, que me concedeu uma bolsa para realizar pesquisas sobre a filosofia de Bertrand Russell na McMaster University, instituição curadora do acervo mencionado.
} 
[12 de fevereiro de 1964] ANEXO I

Dear President Goulart,

I have had in mind some time to write to you in order to ask whether you would consider receiving two of my representatives for the purpose of discussing the work of the recently formed Bertrand Russell Peace Foundation. I have heard of your interest in international affairs and of your concern for the welfare of you people. This has encouraged me to approach you in the hope that you would entertain the possibility of some form of public association with it and its objectives.

I am enclosing a brochure which sets out the aims of the Peace Foundation. These are ambitious aims but, I am confident, they are achievable and essential if our hopes for world peace and the betterment of mankind's circumstances are to be finally established. I look forward to your reply to my request with great interest, and I remain hopeful you will honour our work by your interest in it.

Your sincerely,

Bertrand Russell

\section{A carta a Castelo Branco}

Em 5 de maio de 1964, Russell escreve a Castelo Branco demonstrando preocupação "com a prisão de um grande número de intelectuais e membros da esquerda política” no Brasil. Na carta, ele sugere que Castelo Branco permita “uma anistia aos presos políticos”. Não foram encontradas cartas de autoridades brasileiras em resposta a Russell. 
Dear President Blanco,

I am writing to express concern over the arrest of large numbers of intellectuals and members of the political left in your country. I do not wish to intrude in the domestic affairs of your country, however, I cannot help voicing my concern at the reports of widespread arrest. I hope you will find it possible on humanitarian grounds to permit an amnesty for political prisoners in Brazil.

I wish to appeal as well on behalf of nine Chinese citizens connected with legitimate professional activities in your country. I write out of concern for the good name of Brazil and hope you will find it possible to release all those now imprisoned for political offences.

Your sincerely,

Bertrand Russell

Russell havia sido alertado da prisão e da tortura de nove chineses por Li Chu-Wen, um membro do Chinese People Commitee for World Peace, em uma carta de 21 de abril de 1964. Não há informações sobre como ele teria tomado conhecimento da prisão de intelectuais e políticos brasileiros.

\section{As denúncias que chegaram a Russell}

Entre 1964 e 1966, Russell e a BRPF receberam cartas com denúncias sobre violações de diretos humanos no Brasil durante a ditadura militar. Estão entre os remetentes Alzira Rudge, João Agripino da Costa Dória, Phelippe Nagi Haddad e 
Salomão Tabak. A William Ready Division of Archives and Research Collections da McMaster University, responsável pelas questões legais do Bertrand Russell Archives, concedeu os direitos de publicação das cartas assinadas por Russell e pela BRPF, mas informou que não dispõe dos direitos dos outros remetentes, que pertencem aos próprios. Assim, com a difusão desse documento, seria um acontecimento feliz se familiares dos nomes citados, no interesse do conhecimento de novos fatos sobre a história recente do Brasil, entrassem em contato para autorizar a publicação das cartas na íntegra.

Alzira Rudge - Há uma carta de Alzira Rudge, de 20 de janeiro de 1966, em nome da Comissão Feminina de Intercâmbio e Amizade, cuja destinação não é especificada. Parece ser uma carta-manifesto destinada a um grande público, na qual são denunciadas as arbitrariedades do poder judiciário e as prisões de políticos, líderes sindicais, professores universitários e estudantes, com alguns nomes discriminados. Foi escrita em português e não há registro de respostas.

João Agripino da Costa Dória - Entre 1964 e 1965, João Agripino da Costa Dória trocou algumas cartas com Russell e, principalmente, com Ralph Schoenman, que era um dos diretores da BRPF. O conteúdo de duas cartas de João Dória, uma a Schoenman e a outra a Russell, ambas datadas em 3 de outubro de 1964, sugere que ele visitou Russell em sua casa, no País de Gales, para discutir a situação política do Brasil. No encontro, ficou acertado que a BRPF enviaria um observador ao Brasil para avaliar a situação do país e preparar um relatório imparcial a ser enviado às Nações Unidas e à Comissão Internacional de Juristas. Em data desconhecida (provavelmente não muito depois do dia 3 de outubro de 1964), Schoenman diz a patrocinadores (os nomes legíveis são Julius Nyerere, S. Radhakriahnan e Zakir Husain) que a BRPF está "profundamente alarmada com a violação antidemocrática das liberdades humanas no Brasil” e que deseja enviar um observador ao país. Schoenman solicita o financiamento das passagens aéreas e dos custos da missão. Não há informações que atestem se o financiamento foi concedido e se o observador foi, de fato, enviado ao Brasil. 
[Data desconhecida] ANEXO ॥

\author{
Pres. Julius Nyerere \\ Pres. S. Radhakriahnan \\ Vice-Pres. Zakir Husein
}

This is to affirm the Bertrand Russell Peace Foundation, deeply alarmed at the undemocratic violation of human liberty in Brazil, wishes to send an observer to Brazil to assess the present situation and to prepare an impartial report for submissions before the United Nations and the International Commission of Jurists. After discussion with Federal Deputy Doria, we wish to make clear that we are ready to send such an observer, but require the provision of his air fare and costs while in Brazil. If this can be provided, the BRPF puts its facilities at the disposal of those concerned for individual liberty and justice in Brazil.

Ralph Schoenman

Director

Uma carta de Schoenman a João Dória, em 26 de outubro de 1964, mostra que a BRPF estava "fazendo um projeto de pesquisa sobre o Brasil" e que buscava informações sobre a "dominação Americana" e a situação política geral do país.

Phelippe Nagi Haddad - Há uma carta de 19 de maio de 1965 em que Phelippe Nagi Haddad pede a Russell que ele pergunte às Nações Unidades por que Jânio Quadros foi privado de seus direitos políticos por dez anos. Ele cita uma carta que foi assinada por trinta e cinco mil estudantes da USP e enviada ao governo brasileiro solicitando que Jânio tivesse os seus direitos políticos devolvidos, uma vez que ele não havia sido condenado em nenhum crime. Phelippe Haddad diz que pensou em escrever diretamente às Nações Unidas, 
mas que achava que o seu apelo pessoal não teria efeito contra as ações do governo. Por isso, ele pediu a Russell que o ajudasse com a questão. Em 9 de junho de 1965, a BRPF, representada por Pamela Wood, envia uma carta à Anistia Internacional.

Dear Sirs,

We have received a letter from a Brazilian who states that President Janio Quadros has been deprived of his political rights for ten years. The letter refers to the fact that a letter signed by 35.000 university students from the State of Sao Paulo was recently sent to the Brazilian Government, requesting that Janio's rights be given back to him. Since he was found innocent of any crime.

We should be grateful if you could provide us with further details concerning President Janio Quadros. We look forward to hearing from you.

Yours sincerely,

Pamela Wood

A Anistia Internacional, que tinha status consultivo das Nações Unidas e do Conselho Europeu, respondeu a carta da BRPF no dia 25 de junho de 1965. Andre Mann, do Escritório de Investigação, diz que, apesar de eles estarem acompanhando a situação no Brasil muito de perto, não haviam recebido nenhuma informação recente sobre Jânio Quadros. Sabiam apenas que ele havia sido "expurgado", através de uma matéria publicada no New York Times em 12 de maio de 1964. A última informação recebida pela Anistia Internacional foi em 28 de outubro de 1964: Jânio Quadros estava sob prisão domiciliar. Por fim, Andre 
Mann diz que ficou sabendo, no início da semana, que Jânio Quadros havia acabado de deixar o Brasil após uma breve estada e que ele possivelmente se encontrava na Europa.

Salomão Tabak - Há três cartas de Salomão Tabak. A primeira foi enviada a Russell no dia 30 de janeiro de 1966, na qual ele conta um episódio que aconteceu no Rio de Janeiro durante a realização da "Conferência Interamericana": cinco conhecidos escritores e jornalistas foram presos. Após as prisões, trezentos intelectuais, escritores, artistas e cientistas assinaram uma declaração solicitando que o governo os liberasse. O governo processou todos eles. Num segundo encontro, outros quatrocentos assinaram um manifesto em defesa dos cinco primeiros e em solidariedade aos trezentos. Os cinco foram soltos, mas, ainda assim, outras pessoas foram presas. Salomão Tabak diz a Russell que "a polícia e a inteligência do exército prendem e torturam pessoas todos os dias". Quem responde a carta é Pamela Wood, em 4 de fevereiro de 1966. Ela solicita mais informações sobre as pessoas que foram presas no Brasil "em virtude de sua oposição ao regime".

[4 de fevereiro de 1966] ANEXO V

Dear Mr. Tabak

Thank you very much for your recent letter to Lord Russell. We should be very interested to receive further information concerning the people arrested in Brasil recently because of their opposition to the regime. It would help us if you could provide us with this information in English, but if you are unable to do this we shall endeavour to have it translated.

With best wishes.

Yours sincerely, 
Salomão Tabak, então, envia um dossiê a Wood, no dia 10 de fevereiro de 1966, com uma lista de dezessete notícias de jornais brasileiros, descritas e comentadas em inglês, comprovando as alegações por ele feitas na primeira carta e denunciando outras violências da ditadura. Logo depois, no dia 13 de fevereiro de 1966, ele envia outra carta a Wood, somando mais duas notícias. O dossiê anexa fragmentos dos jornais da época. Dentre os anexos, estão: Jornal do Brasil, 24 de novembro de 1965 - "IPM indicia intelectuais solidários"; Correio da Manhã, 30 de novembro de 1965 - "IPM solta 8 e poeta sai hoje"; Jornal do Brasil, 19 de outubro de 1965 - "Professores de Brasília renunciam em protesto contra cancelamento de bolsas"; Folha da Semana, 19 de janeiro de 1966 - "SP e Minas: militares condenaram 17 líderes sindicais a 183 anos"; Correio da Manhã, 1 de fevereiro de 1966 - "Presidente baixa AC-7 sem regular eleição".

\section{Bertrand Russell Archives, uma fonte rica, porém desconhecida, de documentos inéditos sobre as ditaduras na América Latina}

Até os últimos dias de seus 97 anos, Russell militou fervorosamente contra a violação dos direitos humanos em todo o mundo. Há mais de uma centena de cartas no acervo que mostram como ele buscou mobilizar a opinião pública internacional contra as ditaduras na América Latina, investigando e denunciando a influência dos Estados Unidos nesses regimes e, muitas vezes, entrando em contato com autoridades dos países envolvidos. Recomendo aos pesquisadores interessados que visitem o catálogo digital completo no endereço eletrônico <http://bracers.mcmaster.ca>, a partir do qual é possível solicitar ao acervo algumas cartas na íntegra, em versão PDF. 


\section{Referências}

RUSSELL, Bertrand. Carta, 12 de fevereiro de 1964, para João Goulart, Bertrand Russell Archives, Collection code RA2, Class no. 320, Document no. 177131, Box no. 9.42. (ANEXO I)

RUSSELL, Bertrand. Carta, 5 de maio de 1964, para Castelo Branco, Bertrand Russell Archives, Collection code RA2, Class no. 320, Document no. 177133, Box no. 9.42. (ANEXO II)

SCHOENMAN, Ralph. Carta, 1964 (data desconhecida), para Pres. Julius Nyerere, Pres. S. Radhakriahnan, Vice-Pres. Zakir Husein e outros (patrocinadores da Bertrand Russell Peace Foundation), Bertrand Russell Archives, Collection code RA2, Class no. 330, Document no. 185098, Box no. 9.57. (ANEXO III)

WOOD, Pamela. Carta, 9 de junho de 1965, para Amnesty International, Bertrand Russell Archives, Collection code RA2, Class no. 330, Document no. 185073, Box no. 9.57. (ANEXO IV)

WOOD, Pamela. Carta, 4 de fevereiro de 1966, para Salomão Tabak, Bertrand Russell Archives, Collection code RA2, Class no. 330, Document no. 185096, Box no. 9.57. (ANEXO V)

TOSI, Giuseppe (org.); FERREIRA, Lúcia de Fátima Guerra (org.). Brasil, violação dos direitos humanos - Tribunal Russell II. João Pessoa: Editora UFPB, 2014. Disponível em: <http://www.justica.gov.br/central-deconteudo/anistia/anexos/brasil_violacao-direitos-humanos-miolo-final.pdf> 


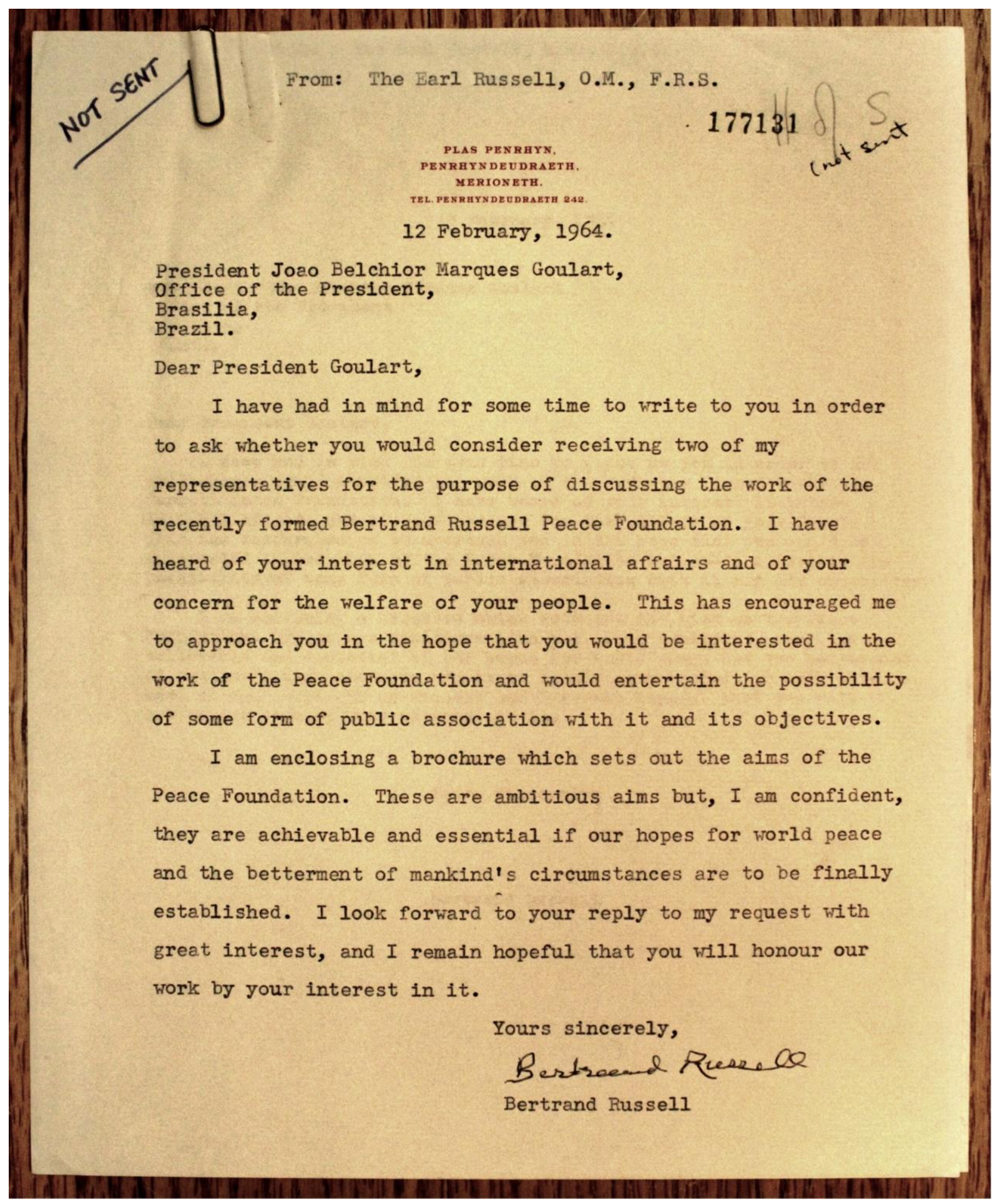




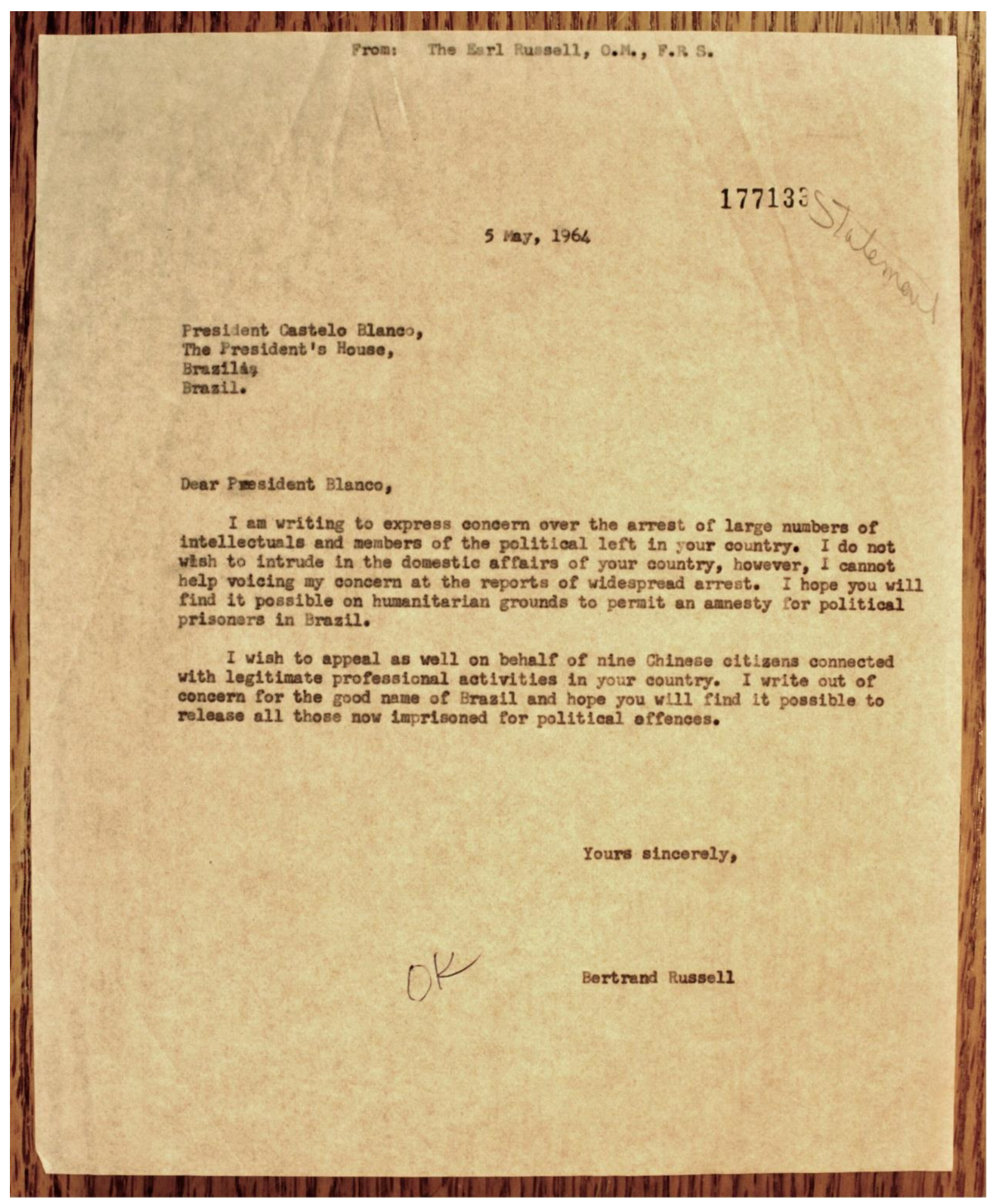




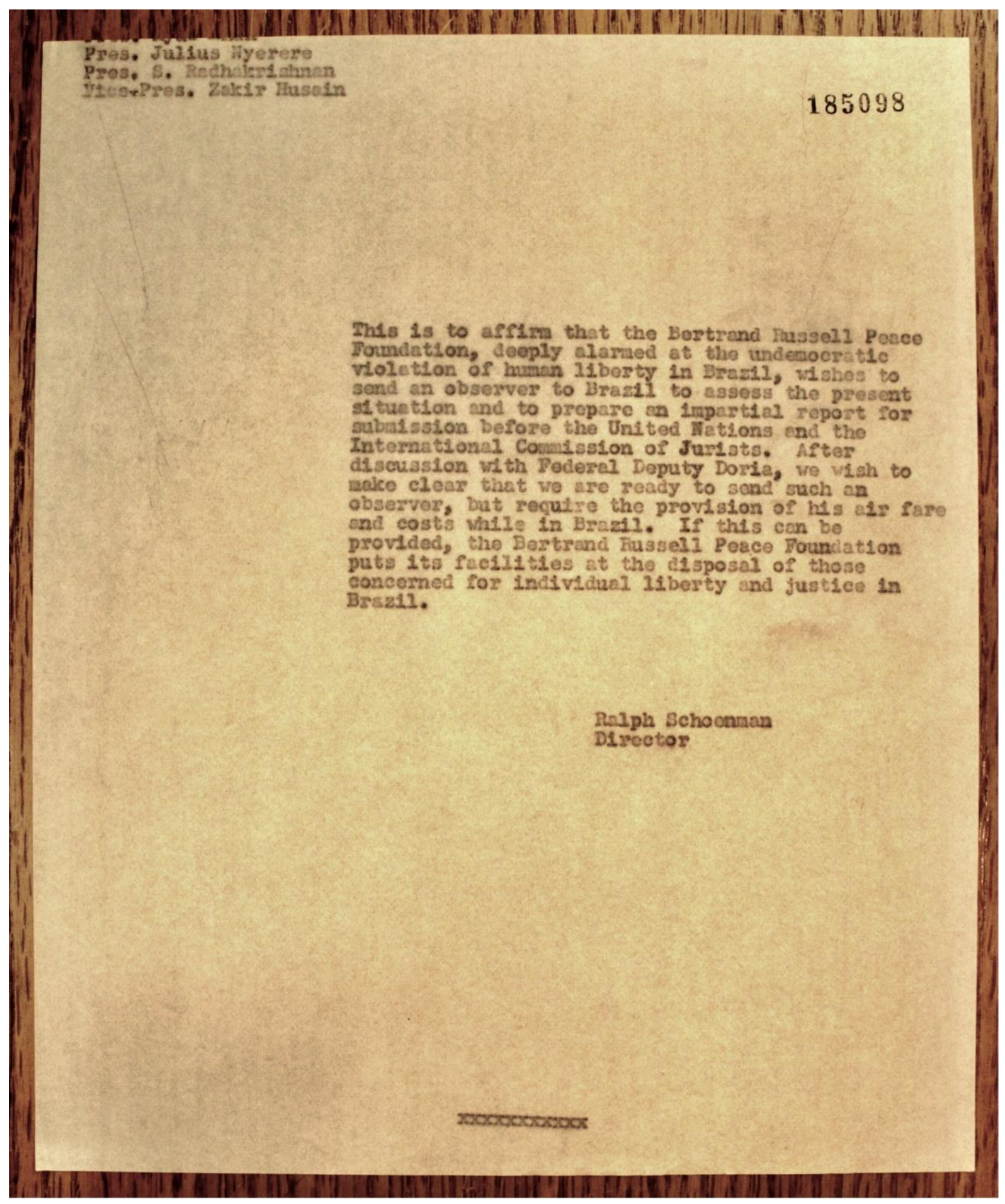




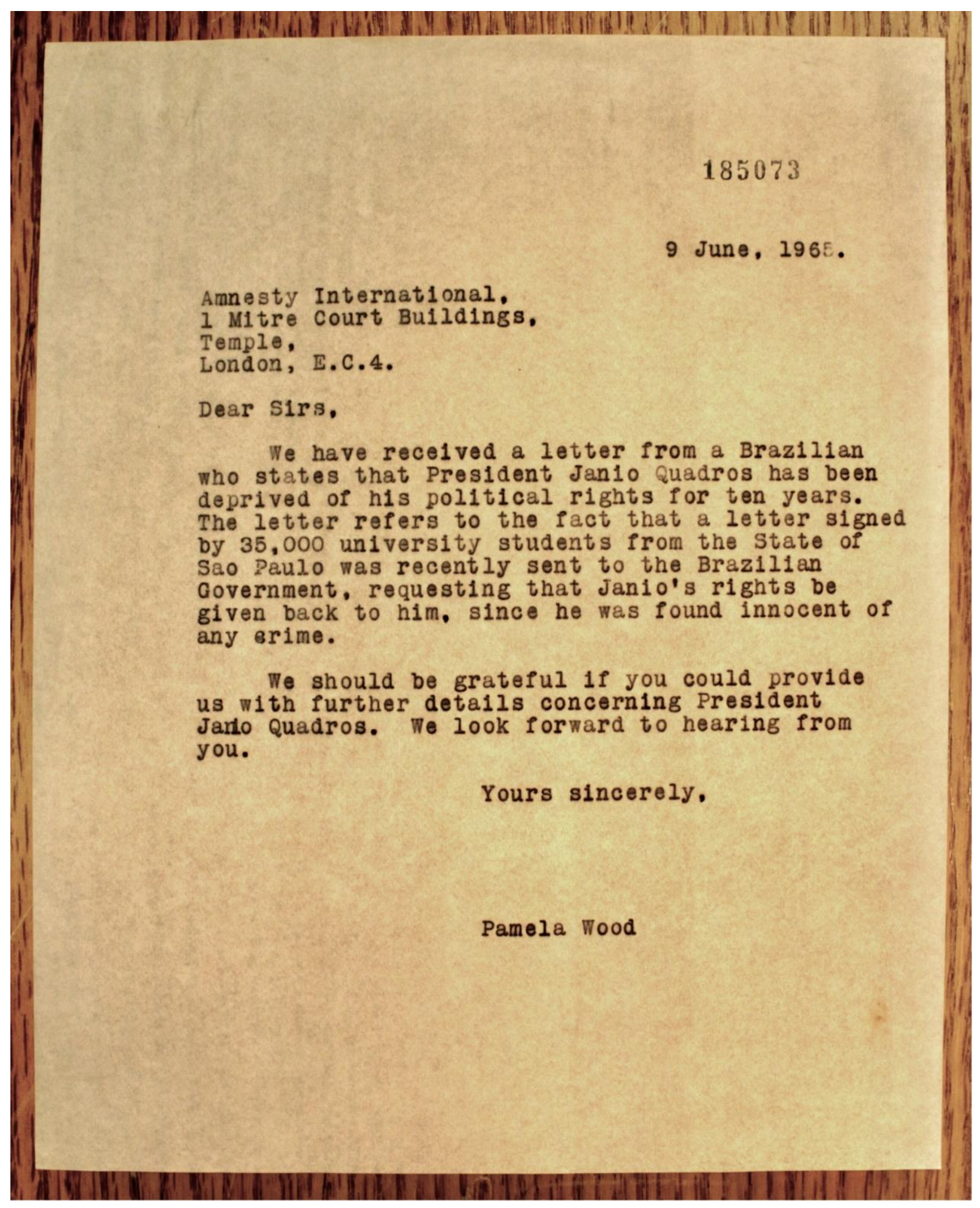




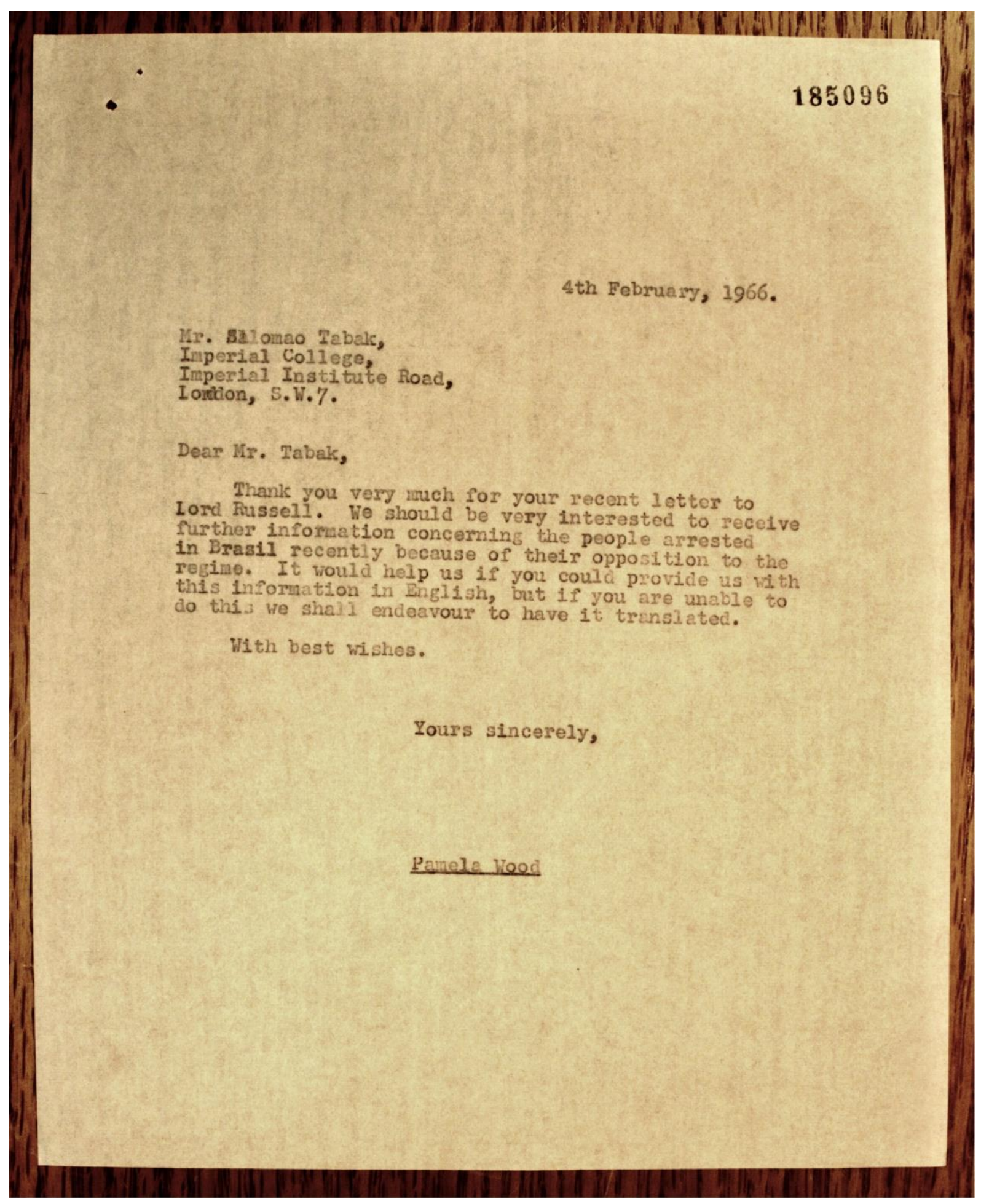

